\title{
PLGA-PEG 共聚物负载多西紫杉醇的药物输运体系的计算机模拟
}

\author{
刘红艳郭泓雨周健* \\ (华南理工大学化学与化工学院 广东省绿色化学产品技术重点实验室 广州 510640)
}

\begin{abstract}
摘要 采用耗散粒子动力学模拟的方法研究了抗癌药物输运体系多西紫杉醇与聚乙丙交酯与聚乙二醇的共聚物 (PLGA-PEG)的自组装形态, 考察了共聚物浓度、共聚物组成和药物含量等对自组装形态的影响. 模拟结果表明, 不同 浓度的 PLGA-PEG 能够和多西紫杉醇自组装成球状、柱状、层状等结构; 一定的浓度下, 亲水的 PEG 嵌段将疏水的 PLGA 嵌段包裹起来形成核壳结构, 可用于疏水药物输运应用. 在比较低的浓度下, 不同组成的 PLGA-PEG 均会形成 球状核壳结构, PEG 嵌段较多时壳层较厚核尺寸较小, PLGA 嵌段较多时核的尺寸较大但壳层较薄, 综合考虑载药量和 稳定性, 模拟结果中 PEG 嵌段的摩尔分数为 $60 \%$ 即 $\mathrm{PLGA}_{40}-\mathrm{PEG}_{60}$ 作为载体时性能较佳. 药物的含量对自组装结构也 有影响, 药物含量较小时形成球状结构, 药物含量较大时, 则会形成柱状结构. 对 $\mathrm{PLGA}_{40}-\mathrm{PEG}_{60}$ 体系, 模拟结果显示 药物、聚合物和水的最佳配比为 $5: 10: 90$. 本工作可为共聚物载药体系的设计与开发提供参考.

关键词＼cjkstart药物输运体系；计算机模拟；自组装；嵌段共聚物；核壳结构
\end{abstract}

\section{Computer Simulations on the Anticancer Drug Delivery System of Docetaxel and PLGA-PEG Copolymer}

\author{
Liu, Hongyan Guo, Hongyu Zhou, Jian* \\ (School of Chemistry and Chemical Engineering, Guangdong Provincial Key Lab for Green Chemical Product Technology, \\ South China University of Technology, Guangzhou 510640)
}

\begin{abstract}
Molecular dynamics (MD) and dissipative particle dynamics (DPD) simulations were integrated to study the morphologies of the drug delivery system self-assembled by amphiphilic block copolymer poly(lactic-co-glycolic acid)-b-poly(ethylene glycol) (PLGA-PEG) and anticancer drug docetaxel. In this work, the solubility parameters used in DPD simulations were calculated by MD simulations. In DPD simulations, the effects of copolymer concentration, copolymer composition and drug content on self-assembled morphologies were investigated. Simulation results show that the morphologies self-assembled by PLGA-PEG and docetaxel (Dtx) undergo the transition from spherical to cylindrical and finally to lamellar micelles when increasing the copolymer concentration from $10 \%$ to $50 \%$ while maintaining the mass ratio of copolymer to drug as $5: 1$. In all cases, core-shell structures were obtained with the hydrophobic PLGA as the core and the hydrophilic PEG as the shell. It is found that PLGA-PEG copolymer with the composition of PEG block ranging from $10 \%$ to $90 \%$ (the total number of the blocks of PLGA-PEG is kept as 100) self-assembles into spherical core-shell structures in aqueous solutions without drug when the copolymer concentration is $10 \%$. When the mole fraction of PEG in PLGA-PEG copolymer is less than $20 \%$, PEG is unable to pack PLGA completely. With the increase of mole fraction of PEG, the PEG shell becomes thicker and the size of the core becomes smaller. In order to moderate the micelle's drug loading efficiency (a small PLGA core will load small amount of hydrophobic anticancer drug Dtx) and stability (a thin PEG shell may make the core-shell micelle unstable), PLGA-PEG copolymer with the molar ratio of PEG to PLGA as $40: 60$ is considered to be the best drug carrier candidate. Besides, the simulation results show that the content of drugs also affects the self-assembled structure. When the drug content is under a certain value, spherical core-shell structures are gained and the size of them grows up with the increase of the drug content. When the drug content is above that value, the spherical structures will connect each other to form cylindrical structures. Considering the advantage of spherical core-shell structures in drug delivery, the optimal mass ratio of docetaxel, $\mathrm{PLGA}_{40}-\mathrm{PEG}_{60}$ copolymer and water is $5: 10: 90$. This work is expected to provide some guidance for the design and development of drug delivery systems.

Keywords drug delivery system; computer simulation; self-assembly; block copolymer; core-shell structure
\end{abstract}

\section{1 引言}

多西紫杉醇 $(\text { docetaxel, Dtx })^{[1]}$ 是属于紫杉醇类的新
一代抗癌药物, 它能够作为微管解聚抑制剂使细胞分裂 停止于有丝分裂期从而抑制细胞的增殖. 它的功效约是

*E-mail: jianzhou@scut.edu.cn; Tel.: 0086-020-87114069

Received August 3, 2012; published October 25, 2012.

Project supported by Program for New Century Excellent Talents in Universities, Ministry of Education, China (No. NCET-07-0313), National Natural Science Foundation of China (No. 20876052) and Guangdong Natural Science Foundation (No. S2011010002078).

项目受教育部新世纪优秀人才支持计划(No. NCET-07-0313)、国家自然科学基金(No. 20876052)及广东省自然科学基金(No. S2011010002078)资助. 
紫杉醇的 2 倍 $^{[2]}$, 其在细胞内的浓度比紫杉醇的高约 3 倍, 在细胞内滞留时间也更长, 已广泛用于治疗卵巢 癌、乳腺细胞癌和非小细胞癌等. 多西紫杉醇在水中溶 解度极小 (约 $10 \mathrm{mg} / \mathrm{L})^{[3]}$, 目前临床使用的制剂采用吐温 80 为增溶剂, 吐温 80 具有溶血性, 大多数患者均出现 过敏反应. 此外, 多西紫杉醇最主要的剂量限制性毒性 反应是使中性粒细胞减少, 且白细胞减少呈剂量依赖性, 药物在血浆中的浓度就直接决定着毒性发生的可能性 及严重程度 ${ }^{[4]}$. 因此如果采用常规静脉注射方法给药, 不仅存在副反应，而且由于药物停留时间问题需每三周 给药一次. 通过载体来对药物进行控制释放则可以尽量 减小药物对人体的副作用, 从而提高药物的治疗效果. 两亲性嵌段共聚物能自组装形成胶束结构, 它们作为一 类有前景的药物控制释放体系, 已受到研究者的青 睐 $^{[5 \sim 9]}$. 在水溶液中, 两亲性共聚物会自组装形成具有 疏水内核和亲水外壳的核壳结构胶束, 因此采用两亲性 嵌段共聚物作为药物载体颇具优势.

聚乙丙交酯共聚物 [poly(lactic-co-glycolic acid), PLGA] 具有良好的生物相容性、生物降解性、无毒 ${ }^{[10]}$. 通 过调整共聚比还可调控 PLGA 的降解速度, 据实验研 究 $^{[11]}$, 当两种单体的共聚比为 50/50 时, 降解速度最快. 由于聚酯结构具有疏水性, 因而可用作两亲性嵌段共聚 物的疏水核. 载药胶束在体内的稳定性是载药体系应用 到人体中的一大挑战. 聚乙二醇 (polyethylene glycol, $P E G)^{[12]}$ 不仅具有亲水、无毒、生物相容等性质，还被认 为具有抗生物污染 ${ }^{[13 ~ 15]}$ 即阻抗蛋白质吸附的特性, 作 为两亲性嵌段共聚物的亲水嵌段时, 能够维持共聚物形 成胶束的稳定性, 常可用作两亲性嵌段共聚物胶束的壳. Gref 等 ${ }^{[16]}$ 证明了 PEG 形成的嵌段共聚物能够显著提高 聚合物纳米粒子在体内的循环时间. PEG 壳能够有效地 阻抗血液中蛋白、细胞的粘附从而增强胶束在血液中的 稳定性、延长体内的停留时间, 并且能形成有利于进入 肿瘤血管系统的纳米级尺寸. 因此, 具有 PEG 壳的嵌段 共聚物广泛用作抗癌药物的载体 ${ }^{[17 ~ 20]}$. 目前, PEG 和 PLGA 均已被美国食品药品管理局(FDA)许可用于药物 传输 $[21,22]$.

PLGA-PEG 是实验载药研究中较常见的一个体系, 已有实验制备了 PLGA-PEG 嵌段共聚物并对其药物传 输性能进行了研究, 主要包括载体的稳定性、对药物的 包载及释放以及靶向性改性等. Gref 等 ${ }^{[16]}$ 制备了 PEG 分 子量不同的 PLGA-PEG 嵌段共聚物, 可观察到随着 PEG 分子量的提高 PEG 壳增厚, 当 PEG 分子量为 5000 时, 其对蛋白质的吸附量最小, 其在体内的循环时间也 延长. Mallarde 等 ${ }^{[23]}$ 制备了不同分子量和不同 PEG 含量 的 PLGA-PEG 嵌段共聚物, 它们能够通过 PLGA 的降解 释放高度疏水的十肽激素药物替维瑞克, 且降解过程中 自组装结构的尺寸保持不变. Davaran 等 ${ }^{[24]}$ 制备了不同 组成的 PLGA-PEG 共聚物, 它们能够自组装成尺寸为
60 100 nm 的粒子, 这些粒子对阿霉素(ADR)的包封率 为 $25 \% \sim 30 \%$. Mo 等 ${ }^{[25]}$ 用 PLGA-PEG 封装 $A D R$, 用 SEM 观察得到 200 $230 \mathrm{~nm}$ 的球型光滑均匀纳米粒子, ADR 的包封率为 $30 \% \sim 35 \%$, 对小鼠静脉给药后，药物 停留时间有效延长. Pamujula 等 ${ }^{[26]}$ 制备了 PEG 含量为 $5 \% \sim 15 \%$ 的 PLGA-PEG, 它们均能形成光滑的球型粒 子, 其中 PEG 含量为 $15 \%$ 的 PLGA-PEG 被 4T1 细胞摄 取的量比其他的都要高, 他们推测可能原因是它的尺寸 最小、zeta 电势最低. Ashjari 等 ${ }^{[27]}$ 将胰岛素封装到 PLGA-PEG 胶束中, 载药量达 $3.9 \mathrm{wt} \%$, 包封率为 55 $\mathrm{wt} \%$ ，药物能够持续释放而且没有观察到突释现象.

目前, 对药物传输体系的研究方法主要是通过实验 大量地合成载体材料、反复地探索来获得较好的配方组 成，这会造成原料以及时间的大量消耗，成本较高. 现 有的实验技术还很难用来研究药物传输体系的结构一性 能关系, 如药物在载药微粒中的分布状况、载药微粒形 成的机理、药物释放的机理等. 采用计算机模拟方法则 可弥补这些不足，而且还能节约时间、节约成本，共聚 物的自组装行为发生的时间尺度主要在介观范围, 耗散 粒子动力学(Dissipative Particle Dynamics, DPD)就是处 于介观层次的一种计算机模拟方法, 并已用于研究嵌段 共聚物的自组装、相分离、药物组装等现象 ${ }^{[28 ~ 39]}$. Guo 等 ${ }^{[36]}$ 通过 DPD 方法研究了以硝苯地平为模型药物、PLA 为载体、PVA 为稳定剂的药物传递体系的结构性能关系. Posocco 等 ${ }^{[37]}$ 通过介观模拟的方法研究了结构和浓度等 物理因素对水溶液中(D-L)-PLA/PEO 二/三嵌段共聚物 自组装结构的影响, 得到了这些体系的相图, 并预测药 物传递体系的不同形态. Rodriguez-Hidalgo 等 ${ }^{[38]}$ 采用 DPD 模拟研究了聚苯乙烯-二乙烯基苯 $[\mathrm{P}(\mathrm{ST}-\mathrm{DVB})]$ 载 体在酸性环境中对药物的释放机理. Masoud 等 ${ }^{[39]}$ 采用 介观模型探索了在外部刺激下中空微胶囊中纳米粒子 和线型大分子的释放. 本文将采用 DPD 的方法来模拟 嵌段共聚物 PLGA-PEG 和多西紫杉醇在水溶液中的自 组装行为, 考察共聚物组成、共聚物的浓度以及药物的 浓度等因素对自组装体结构的影响, 来加深对两亲性共 聚物载药体系的认识, 以期能够为药物传输体系的设计 与开发提供指导.

\section{DPD 模拟方法}

\section{1 基本原理}

DPD 模拟中采用粗粒化模型，即几个原子划分为 一个珠子, 相邻珠子间通过弹簧相连. DPD 模拟中假设 所有的珠子具有相同的质量和体积. 珠子的运动符合牛 顿运动方程 ${ }^{[40]}$,

$$
\frac{\mathrm{d} \boldsymbol{r}_{i}}{\mathrm{~d} t}=\boldsymbol{v}_{i}, m_{i} \frac{\mathrm{d} \boldsymbol{v}_{i}}{\mathrm{~d} t}=\boldsymbol{f}_{i}
$$

式(1)中, $\boldsymbol{r}_{i}, \boldsymbol{v}_{i}, m_{i}$ 和 $\boldsymbol{f}_{i}$ 分别表示珠子 $i$ 的位置、速度、质 量和总的作用力. 在 DPD 中, 珠子所受到的力 $\boldsymbol{f}_{i}$ 包括保 
守力 $\left(\boldsymbol{F}_{i j}^{\mathrm{C}}\right)$ 、耗散力 $\left(\boldsymbol{F}_{i j}^{\mathrm{D}}\right)$ 和随机力 $\left(\boldsymbol{F}_{i j}^{\mathrm{R}}\right)$, 由式(2)表示,

$$
\boldsymbol{f}_{i}=\sum_{j \neq i}\left(\boldsymbol{F}_{i j}^{\mathrm{C}}+\boldsymbol{F}_{i j}^{\mathrm{D}}+\boldsymbol{F}_{i j}^{\mathrm{R}}\right)
$$

珠子所受的这三种力的大小和方向由珠子的位置和速 度决定. 珠子之间是否存在相互作用由截断半径 $r_{\mathrm{c}}$ 决定, 在 DPD 中 $r_{\mathrm{c}}=1$. 三种力的计算公式如下所示,

$$
\begin{aligned}
& \boldsymbol{F}_{i j}^{\mathrm{C}}= \begin{cases}a_{i j}\left(1-r_{i j}\right) \hat{r}_{i j} & \left(r_{i j}<1\right) \\
0 & \left(r_{i j} \geqslant 1\right)\end{cases} \\
& \boldsymbol{F}_{i j}^{\mathrm{D}}=-\gamma \omega^{\mathrm{D}}\left(r_{i j}\right)\left(\hat{r}_{i j} \cdot \boldsymbol{v}_{i j}\right) \hat{r}_{i j} \\
& \boldsymbol{F}_{i j}^{\mathrm{R}}=\sigma \omega^{\mathrm{R}}\left(r_{i j}\right) \theta_{i j} \hat{\boldsymbol{r}}_{i j}
\end{aligned}
$$

其中, $a_{i j}$ 为珠子 $i$ 和 $j$ 之间的排斥参数, $\boldsymbol{r}_{i j}=\boldsymbol{r}_{i}-\boldsymbol{r}_{j}$, $r_{i j}=\left|\boldsymbol{r}_{i j}\right|, \hat{\boldsymbol{r}}_{i j}=\boldsymbol{r}_{i j} /\left|\boldsymbol{r}_{i j}\right|, \boldsymbol{v}_{i j}=\boldsymbol{v}_{i}-\boldsymbol{v}_{j} ; \sigma$ 为噪声振幅; $\boldsymbol{\gamma}$ 为耗散 力参数; $\theta_{i j}$ 为平均值等于 0 的随机变量; $\omega^{\mathrm{D}}\left(r_{i j}\right)$ 和 $\omega^{\mathrm{R}}\left(r_{i j}\right)$ 为权重函数, 二者之间满足式(6)的关系, 而噪声振幅和 耗散力参数满足式(7)的关系.

$$
\begin{aligned}
& \omega^{\mathrm{D}}\left(r_{i j}\right)=\left[\omega^{\mathrm{R}}\left(r_{i j}\right)\right]^{2}=\left\{\begin{array}{cc}
\left(1-r_{i j}\right)^{2} & \left(r_{i j}<1\right) \\
0 & \left(r_{i j} \geqslant 1\right)
\end{array}\right. \\
& \sigma^{2}=2 \gamma \kappa_{\mathrm{B}} T
\end{aligned}
$$

除了上述三个力之外, 同一分子内相邻珠子间还存 在弹性力 $\boldsymbol{F}_{i j}^{\mathrm{S}}=\sum_{j} C_{\mathrm{S}} r_{i j}$, 其中 $C_{\mathrm{S}}$ 为弹簧常数 ${ }^{[40 \sim 42]}$.

\section{2 模型}

本文以 PLGA-PEG 和多西紫杉醇 Dtx 分别作为聚 合物和药物模型, 图 1 为该体系粗粒化的示意图. 按照 不同的单体, PLGA-PEG 被划分为 3 个珠子, 分别标记 为 $\mathrm{L}, \mathrm{G}$ 和 $\mathrm{E} ; \mathrm{Dtx}$ 被划分为 3 个珠子, 分别标记为 $\mathrm{D} 1, \mathrm{D} 2$, $\mathrm{D} 3 ; 3$ 个水分子划分为 1 个珠子 $\mathrm{W}$, 这样可以很好地再 现水的张力 ${ }^{[43]}$.

\section{3 参数}

在 DPD 模拟中, 很重要的步骤是要获得保守力中 的排斥参数 $a_{i j}$. 当体系的数密度为 3 时, 不同珠子间的 排斥参数满足 $a_{i j}=a_{i i}+3.27 \chi_{i j}{ }^{[41,42]}$. 其中, $a_{i i}$ 为相同珠子 间的 DPD 排斥参数, 存在如下关系 ${ }^{[44]}$,

$$
a_{i i}=k_{\mathrm{B}} T \frac{k^{-1} N_{\mathrm{m}}-1}{2 \alpha \rho_{\mathrm{DPD}}}
$$

其中, 在 DPD 模拟中 $k_{\mathrm{B}} T$ 通常设为 $1, k^{-1}$ 为常温常压下 水的无因次压缩因子, 约为 $15.98 ; N_{\mathrm{m}}$ 是一个 DPD 水珠 子所代表的水分子数; 常数 $\alpha=0.101 \pm 0.001 ; \rho_{\text {DPD }}$ 为体 系的数密度. 故当 1 个粗粒化水珠子表示 3 个水分子时, $a_{i i}=78$.
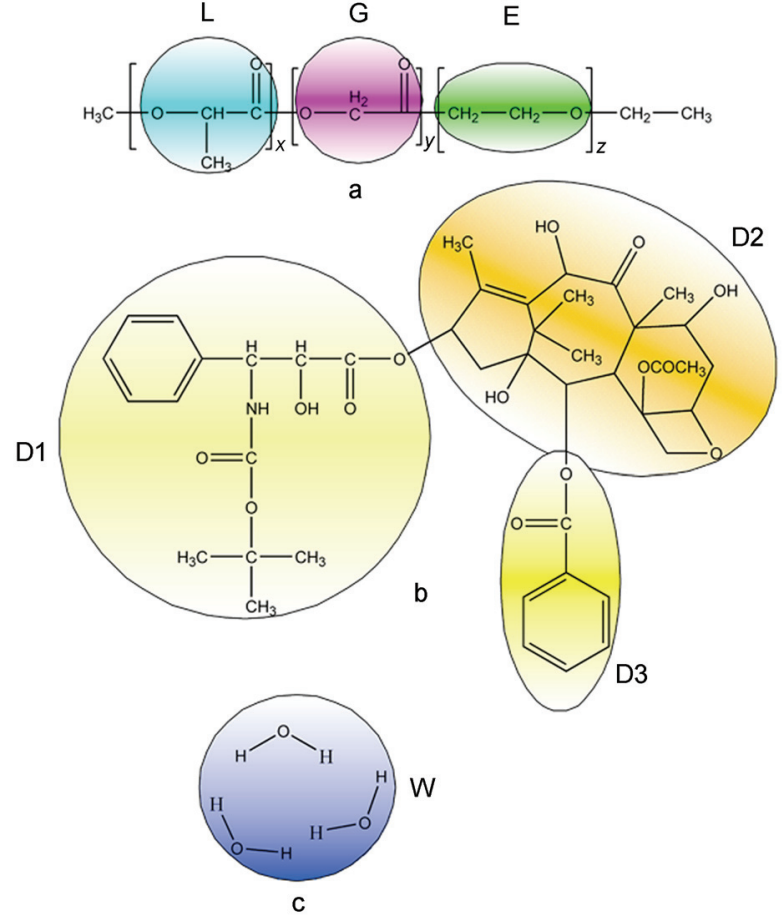

图 1 体系的粗粒化, $\mathrm{a}$ 为 PLGA-PEG 的划分; $\mathrm{b}$ 为 Dtx 的划分; $\mathrm{c}$ 为水 的划分

Figure 1 Coarse graining of the simulated system. a represents PLGA-PEG; $b$ is Dtx and $c$ refers to water

$\chi_{i j}$ 为不同珠子间的 Flory-Huggins 参数, 可由实验获 得, 也可以通过混合能法 ${ }^{[4,45]}$ 、溶解度参数法 ${ }^{[43,46,47]}$ 来 获取. 本文是通过溶解度参数法来获得 $\chi_{i j}$, 即采用 Materials Studio4.4 软件(Accelrys 公司)中的 Amorphous Cell 和 Discover 模块, 选取 COMPASS 力场, 通过分析 内聚能密度得到溶解度参数 $\delta_{i}$, 再通过式(9)即可得到不 同珠子间的 Flory-Huggins 参数 $\chi_{i j}$.

$$
\chi_{i j}=\frac{V}{R T}\left(\delta_{i}-\delta_{j}\right)^{2}
$$

式(9)中 $R$ 为理想气体常数; $\delta_{i}$ 和 $\delta_{j}$ 分别为珠子 $i$ 和珠子 $j$ 的溶解度参数; $T$ 为体系的温度; $V$ 为珠子 $i, j$ 的平均摩尔 体积.

通过上述方法可以获得不同珠子间的排斥参数，如 表 1 所示.

表 1 珠子间的排斥参数

Table 1 Repulsion parameters between beads

\begin{tabular}{lccccccc}
\hline & D1 & D2 & D3 & E & L & G & W \\
\hline D1 & 78.00 & & & & & & \\
D2 & 79.50 & 78.00 & & & & & \\
D3 & 78.02 & 78.87 & 78.00 & & & & \\
E & 81.95 & 88.43 & 80.55 & 78.00 & & & \\
L & 79.65 & 78.15 & 78.83 & 82.96 & 78.00 & & \\
G & 78.98 & 78.01 & 78.45 & 81.76 & 78.04 & 78.00 & \\
W & 222.3 & 288.3 & 161.6 & 114.0 & 161.3 & 149.3 & 78.00 \\
\hline
\end{tabular}


由上表可观察到, D1, D2, D3, L, G 珠子与 W 珠子 间的排斥参数均偏离 78.00 较大, 说明它们与水珠子间 的排斥力较强, 也就是这些珠子的亲水性比较差, 而 $\mathrm{E}$ 珠子与 $\mathrm{W}$ 珠子间的排斥参数小于其它珠子与 $\mathrm{W}$ 珠子间 的排斥参数, 说明与其它珠子相比较, $\mathrm{E}$ 是比较亲水的. 所以，从计算得到的 DPD 排斥参数也可验证由 D1, D2, D3 构成的药物是疏水的, 而由 E, L, G 构成的嵌段共聚 物具有两亲性.

本文进行 DPD 模拟所采用的软件是 Materials Studio 4.4. 模拟盒子大小为 $40 r_{\mathrm{c}} \times 40 r_{\mathrm{c}} \times 40 r_{\mathrm{c}}$, 珠子数密度 $\rho_{\text {DPD }}$ 选为 3 , 体系共含有 192000 个 DPD 珠子, $x, y, z$ 三 个坐标方向上均采用周期性边界条件, 弹簧常数 $C_{\mathrm{S}}$ 设 为 4 , 耗散力参数 $\gamma$ 设为 4.5 , 模拟步数设为 $4 \times 10^{5}$ 步以 保证体系达到平衡. 模拟的时间步长设为 $0.05 \tau$, 其中 $\tau$ 为 DPD 模拟所对应的真实时间单位, $\tau=(14.1 \pm$ $0.1) N_{\mathrm{m}}{ }^{5 / 3}[\mathrm{ps}$. 本模拟体系中, 三个水分子为一个珠子, 而每个水分子的体积为 $0.03 \mathrm{~nm}^{3}$, 也就是说每个珠子的 平均体积为 $0.09 \mathrm{~nm}^{3}$, 由于珠子的数密度为 3 , 通过计 算可得到 $R_{\mathrm{c}}$ 约为 $0.65 \mathrm{~nm}$, 所以模拟体系盒子的边长约 为 $26 \mathrm{~nm}$. 由于本文中 $N_{\mathrm{m}}=3$, 故 $\tau=(88.0 \pm 0.8) \mathrm{ps}$, 由 此可计算出本文的模拟时间约为 $1760 \mathrm{~ns}$.

\section{3 结果与讨论}

对于表面未经靶向配体修饰的胶束, 球状胶束纳米 粒子通过细胞的吞噬作用进入细胞的比率比柱状等的 几率大, 所以本文的研究目的主要是考察何条件下可获 得球状胶束结构 ${ }^{[48]}$. 本文主要研究了共聚物的浓度、共 聚物的组成以及药物的含量对自组装结构的影响, 模拟 过程中 PLGA 中乙交酯和丙交酯的摩尔比固定为 50/50, 为得到多西紫杉醇 PLGA-PEG 载药体系的配方设计提 供参考.

\section{1 共聚物浓度对共聚物-药物自组装结构的影响}

在水溶液中加入一定量的 $\mathrm{PLGA}_{40}-\mathrm{PEG}_{60}$ 和药物, 保证聚合物与药物的质量比为 $5: 1$, 改变聚合物和药 物的量, 观察共聚物自组装结构的变化, 即可获得制备 载药体系的最佳浓度. 图 2 为不同浓度的共聚物在水溶 液中的自组装结构, 此处共聚物的浓度定义为共聚物在 体系中的质量分数, 图 $2 \mathrm{a} \sim 2 \mathrm{f}$ 中共聚物的浓度分别为 $10 \%, 15 \%, 20 \%, 25 \%, 30 \%$ 和 $50 \%$. 当聚合物的浓度为 $10 \%$ (图 2a)时, 共聚物自组装形成表面平滑的球状核壳 结构, 其中 PEG 为壳, PLGA 和药物 Dtx 为核, 随着共聚 物浓度的增加(如图 2b), 胶束的尺寸有所增加, 而且相 邻的球状胶束彼此开始粘连; 在共聚物的浓度增大到 $20 \%$ 的过程中, 球状胶束的尺寸继续增大导致相邻的球 形胶束相互融合形成柱状结构, 此时 PEG 依然为壳, PLGA 和药物处于柱状胶束内部; 当共聚物的浓度为 $25 \%$ 时, 柱状结构的半径增大, 相邻的柱状结构相互融 合, 形成层状孔结构; 共聚物的浓度继续增大(如图 2d),
层状结构上的开孔面积缩小; 聚合物的浓度继续增加到 $50 \%$ 时，层状结构上的孔洞消失，得到均匀的层状结构， PEG 在层状结构的上下两层, 药物与 PLGA 位于层状结 构中间层, 这种层状自组装结构可用作表面药物控释涂 层. 为保证药物载体为球状结构, 因此嵌段共聚物的最 优浓度为 $10 \%$.
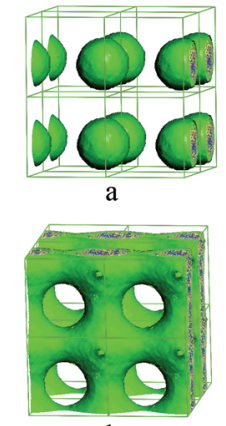

d
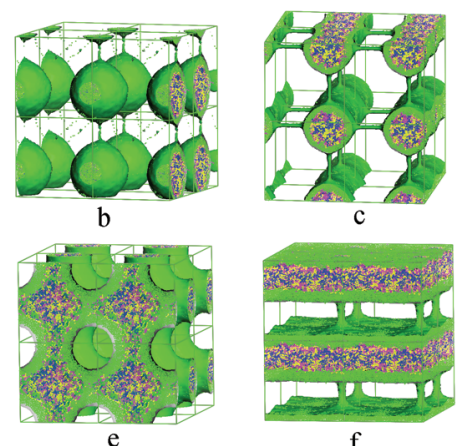

f

$\mathrm{C}-$ represents bead $\mathrm{E}$

bead $\mathrm{G}, \quad$ represents drug

图 2 不同浓度 $P_{L G A}-P_{40}$ F $_{60}$ 嵌段共聚物于水溶液中的形态; $\mathrm{a}=10 \%$, $\mathrm{b}=15 \%, \mathrm{c}=20 \%, \mathrm{~d}=25 \%, \mathrm{e}=30 \%, \mathrm{f}=50 \%$, 其中药物与共聚物的质 量比固定为 $1: 5$

Figure 2 Morphologies of $\mathrm{PLGA}_{40}-\mathrm{PEG}_{60}$ copolymer at different concentrations; $a=10 \%, b=15 \%, c=20 \%, d=25 \%, e=30 \%, f=50 \%$, the mass ratio of copolymer and drug is fixed at $1: 5$

\section{2 共聚物组成对自组装结构的影响}

在共聚物自组装形成的结构中，共聚物的组成会对 其在水溶液中的自组装结构以及稳定性产生影响. 本节 将考察在水溶液中浓度为 $10 \%$ 的不同嵌段比的嵌段共 聚物自组装后的结构, 其中共聚物的重复单元总数保持 为 100 .

如图 3 所示, $\mathrm{a}$ 到 $\mathrm{i}$ 分别为 $\mathrm{PEG}$ 重复单元数为 10,20 , $30,40,50,60,70,80,90$ 的 PLGA-PEG 共聚物的自组装 结构的剖面图. PEG 嵌段的摩尔分数定义为 PEG 的重复 单元数占共聚物重复单元总数的百分数, 由于共聚物重 复单元总数为 100 , 所以图 $3 \mathrm{a}$ 到 $3 \mathrm{i}$ 中 PEG 嵌段的摩尔 分数分别为 $10 \%, 20 \%, 30 \%, 40 \%, 50 \%, 60 \%, 70 \%, 80 \%$ 和 $90 \%$. 当 PEG 嵌段的摩尔分数为 $10 \%$ (图 3a) 和 $20 \%$ (图 3b)时, 可以观察到 PEG 虽然完全在 PLGA 形成的核 外面，但是由于 PEG 的量不足以将大量的 PLGA 完全包 裹, 会有部分 PLGA 直接与水接触, 而不能应用在药物 输运体系中; 由图 $3 \mathrm{a}$ 到 $3 \mathrm{i}$ 可观察到随着 $\mathrm{PEG}$ 含量的增 加, 越来越多的 PEG 包裹在 PLGA 核外面, 当 PEG 嵌 段的摩尔分数为 30\% (图 3c), 40\% (图 3d)时, PLGA 基本 全部被 PEG 包裹, 形成 PEG 为壳、PLGA 为核的核壳 结构, 但此时 PEG 壳的厚度比较薄; 进一步增加 PEG 的含量, PEG 壳层的厚度增加; 当 PEG 嵌段的摩尔分数 为 50\% (图 3e), 60\% (图 3f)时, 形成的结构比较紧凑, 而 且 PEG 壳层均具有一定的厚度, PEG 嵌段摩尔分数为 $60 \%$ 的稍厚一些，疏水药物 Dtx 与疏水链段 PLGA 会通 
过疏水相互作用聚集在核内, 因为加入药物后核的尺寸 会增大, 导致壳层变薄, 因此壳层相对较厚、组成为 60\%的体系在加入药物后体系的稳定性应更强; PEG 摩 尔分数的继续增加会导致 PEG 厚度进一步增大(图 $3 \mathrm{~g}$, $3 \mathrm{~h}, 3 \mathrm{i}$ ), 但是由于链段的总长度是一定的, 也就是 PEG 含量增加, PLGA 的含量就会降低, 即形成的 PEG 壳会 越来越厚而 PLGA 核就会越来越小, 导致它们的载药量 也会越来越小, 这样的组成在实用时是不经济的. 因此, PEG 壳层太厚也是不必要的, 只需在表面覆盖一定厚度 的 PEG 保证胶束的稳定性即可, PEG 链段太长也会造成 PEG 链段的浪费. 为了协调载药量与体系的稳定性, 我 们认为当 PEG 嵌段的摩尔分数为 $60 \%$ 也就是共聚物的 组成为 $\mathrm{PLGA}_{40}-\mathrm{PEG}_{60}$ 时, 所得到的药物载体配方比较 合理.

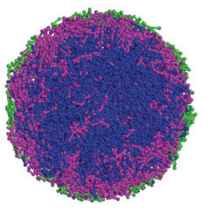

a

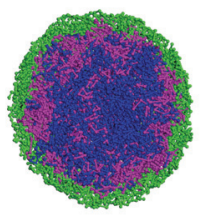

d

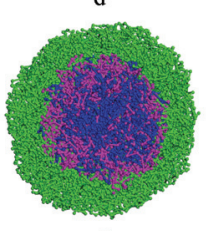

$\mathrm{g}$

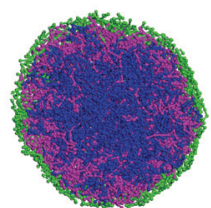

b

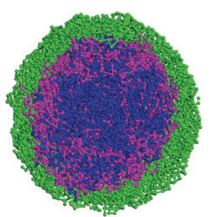

e

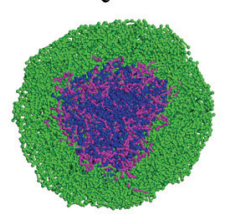

$\mathrm{h}$

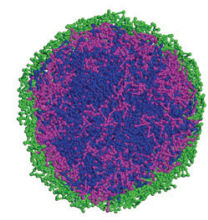

c

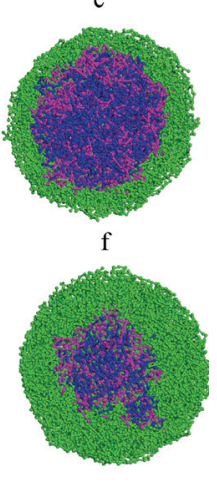

图 3 不同组成的嵌段共聚物自组装结构剖面图, a 到 $\mathrm{i}$ 代表 PEG 的摩 尔分数分别为 $10 \%, 20 \%, 30 \%, 40 \%, 50 \%, 60 \%, 70 \%, 80 \%$ 和 $90 \%$

Figure 3 Sectional views of self-assembled morphologies at different PEG compositions. The molar fraction of PEG from a to $\mathrm{i}$ is $10 \%, 20 \%$, $30 \%, 40 \%, 50 \%, 60 \%, 70 \%, 80 \%$ and $90 \%$, respectively

\section{3 药物含量对共聚物-药物自组装结构的影响}

固定 PLGA-PEG 与水的质量比为 $10: 90$, 加入不 同含量的药物, 观察药物对自组装体系结构的影响, 结 果如图 4 所示.

如图 4a, 当药物：PLGA-PEG：水的质量比为 5： $10 ： 90$ 时, 体系自组装形成了一个球状结构, 其剖面图 如图 5a 右图, 可观察到该球状结构以 PLGA 为核、PEG 为壳, 药物分子均匀地分布在核内; 当药物: PLGA-PEG：水的质量比为 $8: 10: 90$ 时, 如图 4b 所示, 体系依然能够自组装形成球状核壳结构, 但是由于药物 增多, 由 PLGA 和药物构成的核尺寸增大, 使得 PEG 壳 层变薄从而使部分药物、PLGA 出现在球状胶束的表面, 图 5b 右图为该体系的剖面图, 使得体系稳定性下降. 进 一步增加药物的量, 当药物 : PLGA-PEG : 水的质量比

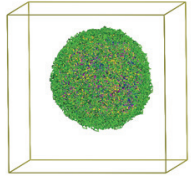

a

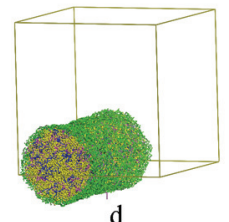

d

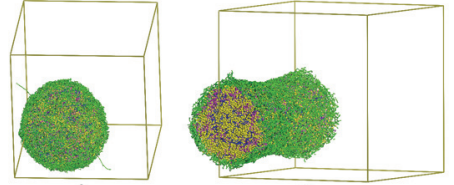

b
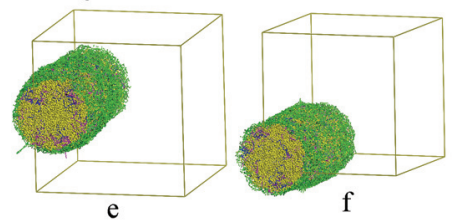

图 4 不同的药物含量对 $\mathrm{PLGA}_{40}-\mathrm{PEG}_{60}$ 自组装结构的影响. 体系中药 物：PLGA-PEG：水的质量比分别为: $\mathrm{a}=5: 10: 90, \mathrm{~b}=8: 10: 90$, $\mathrm{c}=10: 10: 90, \mathrm{~d}=14: 10: 90, \mathrm{e}=18: 10: 90, \mathrm{f}=20: 10: 90$

Figure 4 Morphologies of $\mathrm{PLGA}_{40}-\mathrm{PEG}_{60}$ copolymer with different drug contents. The mass ratio of drug : PLGA-PEG : water: $\mathrm{a}=5: 10$ : $90, \mathrm{~b}=8: 10: 90, \mathrm{c}=10: 10: 90, \mathrm{~d}=14: 10: 90, \mathrm{e}=18: 10: 90$, $\mathrm{f}=20: 10: 90$

为 $10: 10: 90$ 时(图 4c), 由于共聚物和药物的总量比较 大，导致体系不能够自组装形成药物控释领域常用的球 状核壳结构, 而形成了类似哑铃状的结构, 其中 PLGA 和药物依然构成了核部分, PEG 形成壳层保护核. 药物 量继续增加，亚铃状结构逐渐变为柱状结构，核的尺寸 在逐步增加(图 4d, 4e). 当核尺寸增加到一定程度后(图 4f)PEG 壳层不足以完全将柱状结构包裹而导致部分药 物、PLGA 与水接触, 此时的结构如果进入人体，可能 会与血液中的蛋白质疏水微区发生相互作用而粘附在 一起, 对人体健康产生不利的影响. 球状结构比哑铃 状、柱状结构更容易通过内吞作用进入癌细胞从而达到 治疗效果, 因此当 $\mathrm{PLGA}_{40}-\mathrm{PEG}_{60}$ 与水的质量比固定为 $10: 90$ 时, 球状药物传递体系的最大药物质量分数约 为 0.07 (图 4b, 图 5b); 为使 PEG 层具有一定的厚度从 而保证球状药物传递体系的稳定性，最优药物含量则约 为 0.05 (图 4a, 图 5a).
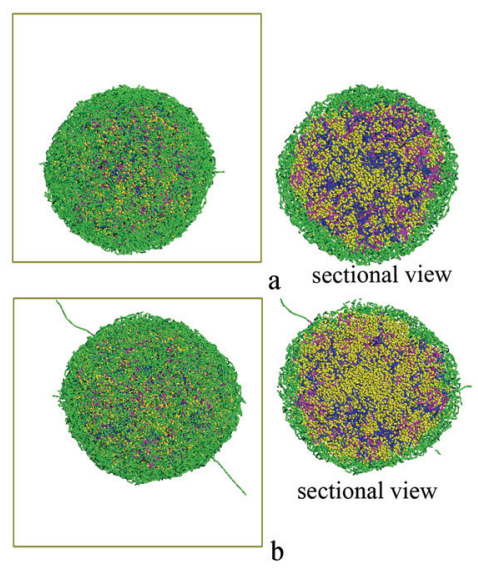

图 5 不同药物含量下球状自组装结构的形态及剖面图. 药物 : PLGA-PEG : 水的质量比分别为: $\mathrm{a}=5: 10: 90, \mathrm{~b}=8: 10: 90$

Figure 5 Spherical morphologies and sectional views of $\mathrm{PLGA}_{40}-\mathrm{PEG}_{60}$ copolymer with different drug contents. The mass ratio of drug: PLGA-PEG : water: $\mathrm{a}=5: 10: 90, \mathrm{~b}=8: 10: 90$ 


\section{4 结论}

本文采用耗散粒子动力学模拟的方法对抗癌药物 多西紫杉醇与共聚物 PLGA-PEG 体系的自组装形态和 体系的配方设计进行了探索. 文中主要考察了共聚物浓 度、共聚物组成和药物含量对自组装形态的影响, 并建 议了较优的配比.

通过模拟可以直观地观察到, PLGA-PEG 共聚物能 够和多西紫杉醇自组装形成核壳结构, 其中疏水的 PLGA 嵌段为核, 亲水的 PEG 嵌段为壳, 多西紫杉醇能 够通过疏水作用进入到 PLGA 核中. 不同的聚合物浓度 会导致 PLGA-PEG 与多西紫杉醇在水溶液中形成不同 的自组装结构, 浓度由低到高会分别形成球状、柱状、 层状孔结构、层状等结构, 这些不同的自组装结构在不 同领域均有应用. 在较低浓度下, 不同组成的 PLGAPEG 共聚物都能形成球状核壳结构. 当 PEG 嵌段的摩 尔分数小于 $20 \%$ 时, PEG 不足以将 PLGA 完全包裹起来; 随着 PEG 嵌段摩尔分数的增加, PEG 壳越来越厚而 PLGA 核的尺寸越来越小, 为了提高载体的载药量且保 证体系以及加入药物后体系在水溶液中的稳定性, 发现 PEG 嵌段的摩尔分数为 $60 \%$ 时的 PLGA-PEG 具有较佳 的综合性能. 药物的含量对自组装结构也有影响, 当加 入的药物比较少时, 体系会形成球状核壳结构, 且在一 定范围内, 随着药物量的增加, 球状结构的尺寸增加. 当药物量超过这个范围时, 随着药物量的增加, 可观察 到球状结构互相连接为亚铃状、柱状. 由上述结果可知, 负载多西紫杉醇的最佳共聚物组成为 $\mathrm{PLGA}_{40}-\mathrm{PEG}_{60}$, 药物、共聚物、水的最优质量比为 $5: 10: 90$. 本文工 作可为药物输运体系的设计与开发提供参考与指导.

\section{References}

[1] Immordino, M. L.; Brusa, P.; Arpicco, S.; Stella, B.; Dosio, F.; Cattel, L. J. Controlled Release 2003, 91(3), 417.

[2] Yang, Y.; Wang, J.-C.; Zhang, X.; Lu, W.-L.; Zhang, Q. J. Controlled Release 2009, 135(2), 175.

[3] Straubinger, R. M.; Balasubramanian, S. V. Methods in Enzymology, Vol. 391, 2005, p. 97.

[4] Chen, Y.; Zhao, Y.; Liu, Q.-Q.; Zou, J. China Pharmacy 2011, 22(2), 130. (陈瑶, 赵银, 刘青青, 邹俊, 中国药房, 2011, 22(2), 130.)

[5] Gaucher, G.; Dufresne, M. H.; Sant, V. P.; Kang, N.; Maysinger, D.; Leroux, J. C. J. Controlled Release 2005, 109(1-3), 169.

[6] Yu, S.-F.; Gu, X.; Wu, G.-L.; Wang, Z.; Wang, Y.-N.; Gao, H.; Ma, J.-B. Acta Chim. Sinica 2012, 70(2), 177. (于树芳, 顾金金, 伍国琳, 王铮, 王亦农, 高辉, 马建标, 化学学报, 2012, 70(2), 177.)

[7] Zheng, X.-L.; Ding, A.-S.; Luo, D.; Gao, H.-S. Acta Chim. Sinica 2012，70(1)，92. (郑兴良，丁爱顺，罗丹，高鸿盛，化学学报， 2012, $70(1), 92$.

[8] Yang, Z.-L.; Li, X.-R.; Yang, K.-W.; Liu, Y. Acta Chim. Sinica 2007, 65(19), 2169. (杨卓理, 李馨汝, 杨可伟, 刘艳, 化学学报, 2007, 65(19), 2169.)

[9] Mao, J.; Gan, Z.-H. Chem. J. Chin. Univ. 2009, 30(11), 2291. (毛静, 甘志华，高等学校化学学报, 2009, 30(11), 2291.).

[10] Tran, V. T.; Karam, J. P.; Garric, X.; Coudane, J.; Benoit, J. P.; Montero-Menei, C. N.; Venier-Julienne, M. C. Eur. J. Pharm. Sci. 2012, $45(1-2), 128$
[11] Vey, E.; Rodger, C.; Meehan, L.; Booth, J.; Claybourn, M.; Miller, A. F.; Saiani, A. Polym. Degrad. Stab. 2012, 97(3), 358.

[12] Alconcel, S. N. S.; Baas, A. S.; Maynard, H. D. Polym. Chem. 2011, 2(7), 1442.

[13] Menz, B.; Knerr, R.; Gopferich, A.; Steinem, C. Biomaterials 2005, 26(20), 4237

[14] Bluemmel, J.; Perschmann, N.; Aydin, D.; Drinjakovic, J.; Surrey, T.; Lopez-Garcia, M.; Kessler, H.; Spatz, J. P. Biomaterials 2007, $28(32), 4739$.

[15] Xie, Y.; Liu, M.-F.; Zhou, J. Appl. Surf. Sci. 2012, $258(20), 8153$.

[16] Gref, R.; Minamitake, Y.; Peracchia, M. T.; Trubetskoy, V.; Torchilin, V.; Langer, R. Science 1994, 263(5153), 1600.

[17] Ukawala, M.; Rajyaguru, T.; Chaudhari, K.; Manjappa, A. S.; Pimple, S.; Babbar, A. K.; Mathur, R.; Mishra, A. K.; Murthy, R. S. R. Drug Delivery 2012, 19(3), 155.

[18] Mothe, C. G.; Azevedo, A. D.; Drumond, W. S.; Wang, S. H.; Sinisterra, R. D. J. Therm. Anal. Calorim. 2011, 106(3), 671.

[19] Li, T.-H.; Han, R.-Y.; Wang, M.-Z.; Liu, C.-B.; Jing, X.-B.; Huang, Y.-B. Macromol. Biosci. 2011, 11(11), 1570.

[20] Zhang, W.-L.; Li, Y.-L.; Liu, L.-X.; Sun, Q.-Q.; Shuai, X.-T.; Zhu, W.; Chen, Y.-M. Biomacromolecules 2010, 11(5), 1331.

[21] Jain, R. A. Biomaterials 2000, 21(23), 2475.

[22] Veronese, F. M.; Pasut, G. Drug Discovery Today 2005, 10(21), 1451.

[23] Mallarde, D.; Boutignon, F.; Moine, F.; Barre, E.; David, S.; Touchet, H.; Ferruti, P.; Deghenghi, R. Int. J. Pharm. 2003 , 261(1-2), 69 .

[24] Davaran, S.; Rashidi, M. R.; Pourabbas, B.; Dadashzadeh, M.; Haghshenas, N. M. Int. J. Nanomed. 2006, 1(4), 535.

[25] Mo, S. M.; Oh, I. J. J. Nanosci. Nanotechnol. 2011, 11(2), 1795.

[26] Pamujula, S.; Hazari, S.; Bolden, G.; Graves, R. A.; Chinta, D. D.; Dash, S.; Kishore, V.; Mandal, T. K. J. Pharm. Pharmacol. 2012, 64(1), 61.

[27] Ashjari, M.; Khoee, S.; Mahdavian, A. R.; Rahmatolahzadeh, R. J. Mater. Sci.: Mater. Med. 2012, 23(4), 943.

[28] Liu, H.-L.; Hu, Y. CIESC J. 2003, 54(4), 440. (刘洪来, 胡英, 化工 学报, 2003, 54(4), 440.)

[29] Feng, J.; Liu, H.-L.; Hu, Y. J. Nanjing Univ. Technol. 2005, 27(2), 105. (冯剑, 刘洪来, 胡英, 南京工业大学学报, 2005, 27(2), 105.)

[30] Xia, J.; Zhong, C.-L. Macromol. Rapid Commun. 2006, 27, 1110.

[31] Zhong, C.-L.; Liu, D.-H. Macromol. Theory Simul. 2007, 16, 141.

[32] Liu, D.-H.; Zhong, C.-L. Polymer 2008, 49(5), 1407.

[33] Xin, J.; Liu, D.-H.; Zhong, C.-L. J. Phys. Chem. B 2009, 113(28), 9364.

[34] Feng, J.; Ge, X.-T.; Shang, Y.-Z.; Zhou, L.-H.; Liu, H.-L.; Hu, Y. Fluid Phase Equilib. 2011, 302, 26.

[35] Guo, H.-Y.; Cui, J.-M.; Sun, D.-L.; Zhou, J. CIESC J. 2012, 11, 3707. (郭泓雨, 崔洁铭, 孙德林, 周健，化工学报，2012，11, 3707.)

[36] Guo, X.-D.; Zhang, L.-J.; Qian, Y.; Zhou, J. Chem. Eng. J. 2007, 131,195

[37] Posocco, P.; Fermeglia, M.; Pricl, S. J. Mater. Chem. 2010, 20, 7742 .

[38] Rodriguez-Hidalgo, M. D. R.; Soto-Figueroa, C.; Vicente, L. Soft Matter 2011, 7, 8224.

[39] Masoud, H.; Alexeev, A. ACS Nano 2012, 6, 212.

[40] Groot, R. D.; Warren, P. B. J. Chem. Phys. 1997, 107(11), 4423.

[41] Groot, R. D.; Madden, T. J. J. Chem. Phys. 1998, 108(20), 8713.

[42] Groot, R. D.; Rabone, K. L. Biophys. J. 2001, $81(2), 725$.

[43] Maiti, A.; McGrother, S. J. Chem. Phys. 2004, $120(3), 1594$.

[44] Sun, D.-L.; Zhou, J. Acta Phys.-Chim. Sin. 2012, 28(4), 909. (孙德 林, 周健, 物理化学学报, 2012, 28(4), 909.)

[45] Zhuang, Q.-X.; Xue, Z.-J.; Liu, X.-Y.; Yuan, Y.-L.; Han, Z.-W. Polym. Compos. 2011, 32(10), 1671.

[46] Zhao, Y.; You, L.-Y.; Lu, Z.-Y.; Sun, C.-C. Polymer 2009, 50(22), 5333 .

[47] Xie, X.-A.; Ding, N.-P.; Liu, H.-B.; Zheng, L.-S.; Xiong, M.-Z. Acta Chim. Sinica 2011, 69(2), 169. (解新安, 丁年平, 刘焕涁, 郑璐丝, 熊明洲, 化学学报, 2011, 69(2), 169.)

[48] Fan, B.-Y.; Fan, Y.-L.; Hu, S.-J.; Sun, L.; Li, M.; Li, Q.; Ji, Y.-B. Chin. Pharm. J. 2011, 46, 1220. (范兵羽, 范玉玲, 胡淑娟, 孙黎, 李永, 李强, 季宇彬, 中国药学杂志, 2011, 46, 1220.) 